\title{
Isolated Mass-Forming IgG4-Related Cholangitis as an Initial Clinical Presentation of Systemic IgG4-Related Disease
}

Seokhwi Kim · Hyunsik Bae Misun Choi · Binnari Kim Jin Seok Heo ${ }^{1}$ Ho Seong Kim ${ }^{2}$ Seung Hee Choi $\cdot$ Kee-Taek Jang

Departments of Pathology and Translational Genomics, 'S Surgery, ${ }^{2}$ Nuclear Medicine, and ${ }^{3}$ Radiology, Samsung Medical Center, Sungkyunkwan University School of Medicine, Seoul, Korea

\author{
Received: November 13, 2015 \\ Revised: November 26, 2015 \\ Accepted: December 1, 2015

\section{Corresponding Author} \\ Kee-Taek Jang, MD \\ Department of Pathology and Translational \\ Genomics, Samsung Medical Center, \\ Sungkyunkwan University School of Medicine, \\ 81 Irwon-ro, Gangnam-gu, Seoul 06351, Korea \\ Tel: +82-2-3410-2763 \\ Fax: +82-2-3410-0025 \\ E-mail: kt12.jang@samsung.com
}

\begin{abstract}
lgG4-related disease (IgG4-RD) may involve multiple organs. Although it usually presents as diffuse organ involvement, localized mass-forming lesions have been occasionally encountered in pancreas. However, the same pattern has been seldom reported in biliary tract. A 61-year-old male showed a hilar bile duct mass with multiple enlarged lymph nodes in imaging studies and he underwent trisectionectomy under impression of cholangiocarcinoma. Gross examination revealed a mass-like lesion around hilar bile duct. Histopathologically, dense lymphoplasmacytic infiltration and storiform fibrosis were identified without evidence of malignancy. Immunohistochemical stain demonstrated rich lgG4-positive plasma cell infiltration. Follow-up imaging studies disclosed multiple enlarged lymph nodes with involvement of pancreas and perisplenic soft tissue. The lesions have been significantly reduced after steroid treatment, which suggests multi-organ involvement of systemic IgG4-RD. Here, we report an unusual localized mass-forming lgG4related cholangitis as an initial presentation of lgG4-RD, which was biliary manifestation of systemic lgG4-related autoimmune disease.
\end{abstract}

Key Words: IgG4-related disease; Bile ducts; Mass-forming; Cholangiocarcinoma
IgG4-related disease (IgG4-RD) may involve multiple organs including pancreas, head and neck region, kidney, lung, retroperitoneum, and lymph node. ${ }^{1}$ Histologically, it is characterized by lymphoplasmacytic infiltration, storiform fibrosis and/ or obliterative phlebitis with IgG4 immunohistochemical reactivity in plasma cells. ${ }^{2}$ In addition to histologic findings, serum IgG4 level is often elevated in patients with IgG4-RD. ${ }^{3,4}$ It is not difficult to diagnose IgG4-RD if an involved organ shows diffuse enlargement with an elevated serum IgG4 level. However, it may not be easy to suspect IgG4-RD if the lesion presents as a localized mass-forming lesion rather than diffuse organ involvement. Although localized mass-forming IgG4-related autoimmune pancreatitis has been reported occasionally, ${ }^{5-7}$ such manifestation in biliary tract has seldom been described in the literature. In addition, most reported biliary cases had simultaneous segmental involvement of bile duct wall as well as massforming lesions. ${ }^{8-10}$ Here, we report a case of localized mass-forming IgG4-related cholangitis, which mimicked hilar cholangio- carcinoma and later progressed to multi-organ involving IgG4related systemic autoimmune disease, with review of relevant literatures.

\section{CASE REPORT}

A 61-year-old male, who had a past medical history of diabetes, coronary artery bypass surgery and idiopathic pulmonary disease, was admitted for the control of his blood sugar level. He complained of general weakness, easy fatigability, and weight loss of $3 \mathrm{~kg}$ for 10 days. Laboratory test results were within normal range except for elevated aspartate aminotransferase (100 U/L), alanine transaminase (114 U/L), and alkaline phosphatase (397 $\mathrm{U} / \mathrm{L})$. Although serum tumor markers were within normal limits ( $\alpha$-fetoprotein, $2 \mathrm{ng} / \mathrm{mL}$; carcinoembryonic antigen, $1.56 \mathrm{ng} /$ $\mathrm{mL}$; carbohydrate antigen 19-9, $13.71 \mathrm{U} / \mathrm{mL}$ ), additional tests for malignancy was conducted due to his weight loss. The magnetic resonance cholangiopancreatography disclosed a mass le- 
sion at hilar bile duct. Magnetic resonance imaging (MRI) revealed a $2.1-\mathrm{cm}$-sized relatively well-demarcated mass at bifurcation of the left hepatic duct. The mass showed high signal intensity in diffusion restriction image and low intensity in apparent diffusion coefficient (ADC) map image, suggestive of type IV hilar cholangiocarcinoma with periductal invasion into underlying hepatic parenchyma (Fig. 1A, B). Since the patient had type 3 variation of intrahepatic bile duct-prior branching of right posterior Glisson pedicle which made the mass to be located apart from the right posterior duct, the lesion was considered to be resectable. Preoperative evaluation of positron emission tomography (PET) showed multiple enlarged lymph nodes in left axillary, common hepatic, portocaval, and aortocaval areas. Ultrasonography-guided biopsy of the axillary lymph node was performed; however, malignant cell was not identified, suggesting a reactive change rather than metastasis. Clinicians decided to perform surgery with an impression of hilar cholangiocarcinoma. A firm mass was detected at upper hilar level dur- ing the operation. Invasion to the left portal vein was suspected and the mass was also close to the right portal vein. Left trisectionectomy was performed for curative resection.

The resected specimen revealed a white firm mass-like lesion, measuring $2.5 \times 2.0 \times 1.3 \mathrm{~cm}$, at bifurcation of the left intrahepatic bile duct (Fig. 1C). Histologic examination showed an extensive infiltration of lymphocytes and plasma cells (Fig. 2A). Storiform fibrosis was noted with a few foci of obliterative phlebitis (Fig. 2B). Immunohistochemical staining for IgG and IgG4 showed numerous IgG- and IgG4-immunoreactive plasma cells (Fig. 2C, D). The IgG4+ plasma cells were identified up to 53 cells per high-power field. IgG4+/IgG plasma cell ratio was 0.42 . According to the consensus statement on the pathology of IgG4-RD, the lesion was diagnosed as "histologically highly suggestive of IgG4-RD." ${ }^{2}$

Following the pathologic diagnosis of IgG4-RD, laboratory tests for serum IgG level was performed and it was within upper normal range in postoperative day $12(1,522 \mathrm{mg} / \mathrm{dL}$; refer-
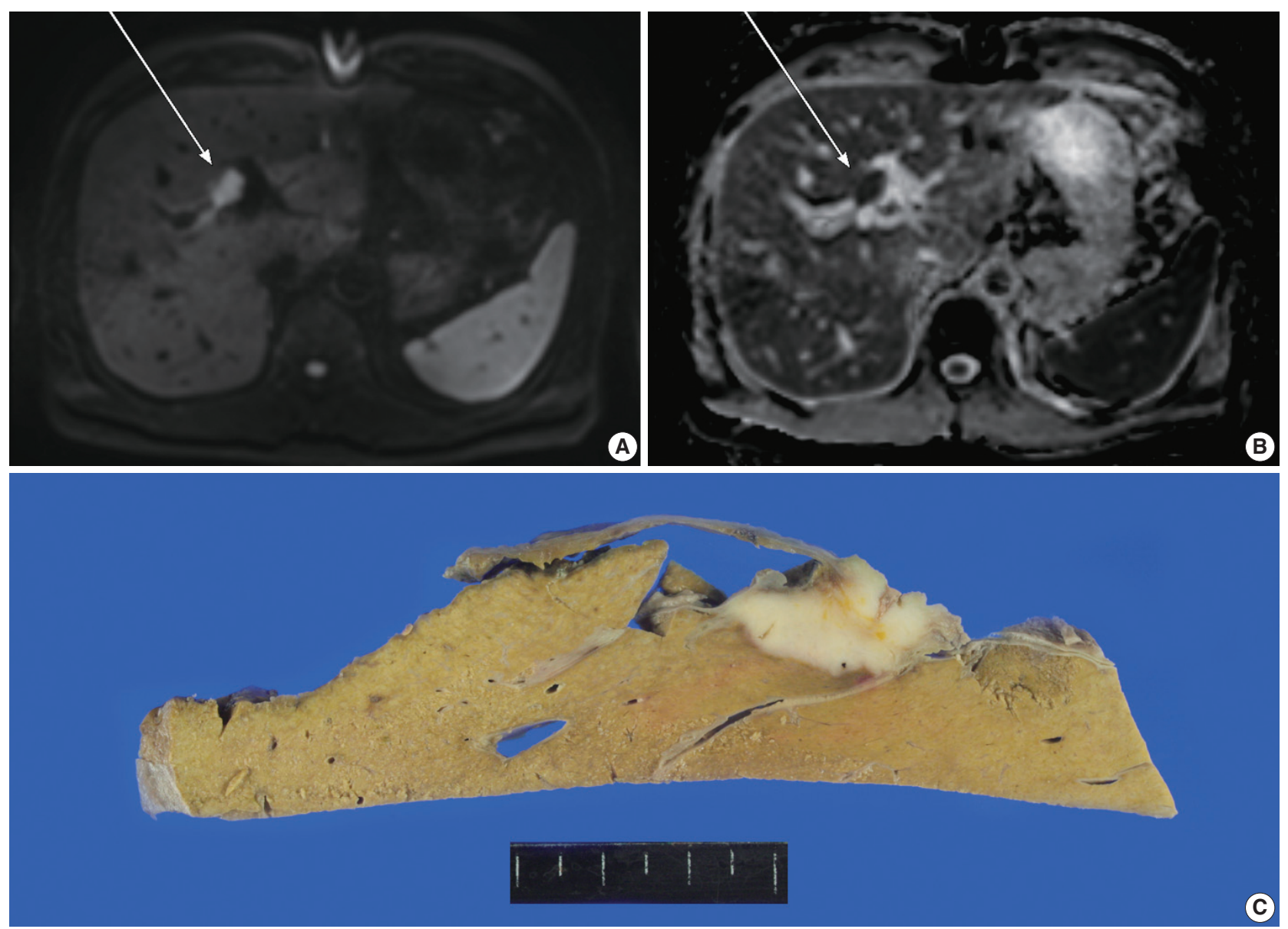

Fig. 1. Magnetic resonance imaging reveals an enhancing mass lesion which has high signal intensity on diffusion restriction phase (A) and low intensity on apparent diffusion coefficient map phase (B), suspicious for malignancy. (C) The cut section of hilar bile duct shows a relatively well-demarcated mass-forming lesion with a hepatic parenchymal invasion. 
ence range, 700 to $1,600 \mathrm{mg} / \mathrm{dL}$ ). All subclasses of $\mathrm{IgG}$ were elevated: $\operatorname{IgG1}, 1,070 \mathrm{mg} / \mathrm{dL}$ (reference range, 341 to $894 \mathrm{mg} / \mathrm{dL}$ ); IgG2, $694 \mathrm{mg} / \mathrm{dL}$ (reference range, 171 to $632 \mathrm{mg} / \mathrm{dL}$ ); IgG3, $134 \mathrm{mg} / \mathrm{dL}$ (reference range, 11.5 to $105.3 \mathrm{mg} / \mathrm{dL}$ ); and $\mathrm{IgG} 4$, $257 \mathrm{mg} / \mathrm{dL}$ (reference range, 2.4 to $121.0 \mathrm{mg} / \mathrm{dL}$ ). Thorough investigation for involvement of other organs by IgG4-RD was done, but it failed to identify any additional abnormality. The patient did not receive corticosteroid treatment because no residual lesion was left after the resection and also because he was diabetic.

After a year, follow-up PET scan revealed an increased fluorodeoxyglucose (FDG) uptake in the biliary trees of right liver, pancreas, and perisplenic soft tissue as well as hypermetabolic lymphadenopathy involving supraclavicular, mediastinal, pulmonary hilar, subcarinal and left abdominal paraaortic lymph nodes, suggestive of progression to extrabiliary multi-organ IgG4-related autoimmune disease (Fig. 3A). Serum IgG level had also increased to $3,677 \mathrm{mg} / \mathrm{dL}$, more than twice as high as the initial value. Fluorescent antinuclear antibody test showed 4+ result. The patient started to take prednisone once a day. However, computed tomography (CT) images after prednisone treatment for 6 months revealed subpleural consolidations and en- largement of mediastinal lymph nodes. As prednisone treatment was continued, the extent of subpleural consolidations diminished on the follow-up CT images. A year persistent treatment markedly decreased FDG uptake in bilateral supraclavicular, left axilla, mediastinal, subcarinal, pulmonary hilar, and retroperitoneal left paraaortic lymph nodes on PET scan, reflecting the patient's multi-organ IgG4-RD response to the steroid (Fig. 3B).

This study was approved by the Institutional Review Board of the Samsung Medical Center (IRB No. 2015-10-203).

\section{DISCUSSION}

IgG4-RD was originally recognized in pancreas as autoimmune pancreatitis. ${ }^{11}$ Concomitant extrapancreatic manifestation is not uncommon in bile duct, lymph node, salivary glands, gastric mucosa, and kidney. ${ }^{1,3,12}$ Solitary organ involvement without pancreatitis has also been reported. ${ }^{1,3,4}$ IgG4-RD tends to present as diffuse lesion in the involved organs, especially in pancreas and biliary tract. ${ }^{13}$ Localized mass-forming autoimmune pancreatitis had frequently been misdiagnosed as malignancy and had resulted in unnecessary surgical resections. ${ }^{6,7}$ In contrast, mass-forming IgG4-related cholangitis is far less common and
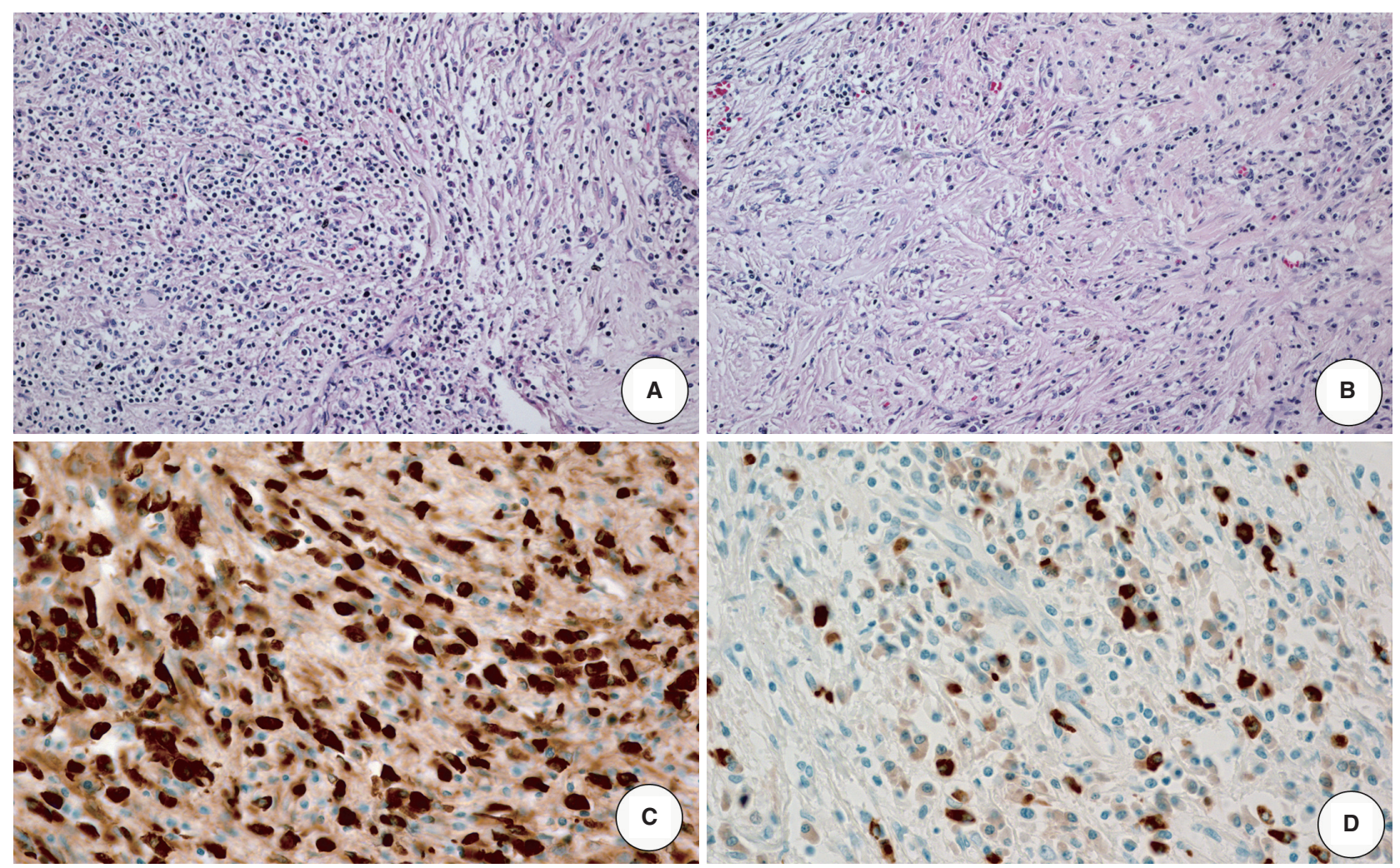

Fig. 2. Histologic examination reveals dense infiltration of lymphocytes and plasma cells (A) and storiform fibrosis (B). Both lgG-immunopositive (C) and lgG4-immunopositive (D) plasma cells are identified by immunohistochemical staining. 

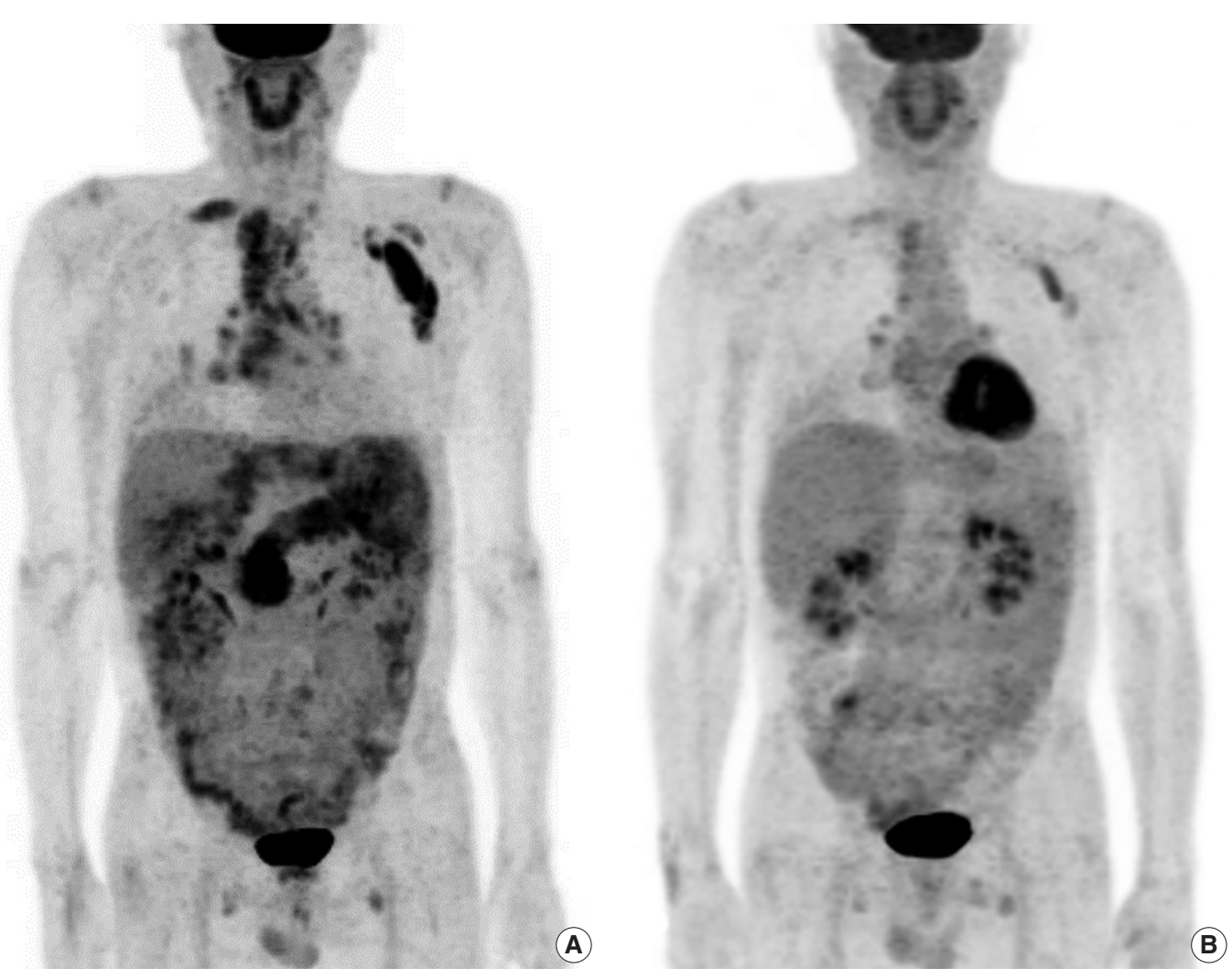

Fig. 3. (A) Fluorodeoxyglucose (FDG) positron emission tomography-computed tomography, maximum intensity projection image show hypermetabolic lesions involving bilateral supraclavicular, left axillar, mediastinal, pulmonary hilar and retroperitoneal lymph nodes, right liver biliary tract, pancreas, and perisplenic area. (B) After steroid treatment, markedly decreased FDG uptake is noted although uptakes in some lymph nodes are still seen.

Table 1. Clinicopathological findings of previously reported cases of mass-forming lgG4-related cholangitis

\begin{tabular}{|c|c|c|c|c|c|c|c|c|c|c|c|}
\hline Reference & Sex & $\begin{array}{l}\text { Age } \\
(y r)\end{array}$ & $\begin{array}{l}\text { Specimen } \\
\text { type }\end{array}$ & Site & Symptom & Radiologic finding & $\begin{array}{l}\operatorname{lgG} 4 \\
\text { count } \\
\text { (/HPF) }\end{array}$ & $\begin{array}{c}\text { Sequence } \\
\text { of biliary } \\
\text { manifestation }\end{array}$ & $\begin{array}{c}\text { Other } \\
\text { manifestations }\end{array}$ & $\begin{array}{l}\text { Serum } \\
\lg G 4 / \lg G\end{array}$ & Follow-up \\
\hline Present case & $\mathrm{M}$ & 61 & Resection & Hilum & $\begin{array}{c}\text { Fatigue, } \\
\text { weight loss }\end{array}$ & Localized mass & 45 & Initial & $\begin{array}{l}\text { Pancreas, } \\
\text { pleura, LN, } \\
\text { perisplenic } \\
\text { soft tissue }\end{array}$ & $\begin{array}{l}257 \\
/ 1,522\end{array}$ & $\begin{array}{l}\text { Response } \\
\text { on steroids }\end{array}$ \\
\hline \multirow[t]{2}{*}{$\begin{array}{l}\text { Deshpande } \\
\text { et al. }{ }^{8}\end{array}$} & M & 68 & Biopsy & $\begin{array}{l}\text { Liver and } \\
\text { IBD }\end{array}$ & Jaundice & $\begin{array}{l}\text { Vague mass with } \\
\text { alternate narrowing } \\
\text { and dilatation of IBD }\end{array}$ & 37 & Initial & $\begin{array}{l}\text { Salivary gland, } \\
\text { retroperitoneum }\end{array}$ & $\begin{array}{l}4,160 \\
/ 3,580\end{array}$ & $\begin{array}{l}\text { Response } \\
\text { on steroids }\end{array}$ \\
\hline & $\mathrm{F}$ & 42 & Resection & Hilum & $\begin{array}{l}\text { Hepatic hilar } \\
\text { mass }\end{array}$ & NA & 140 & Secondary & Pancreas & NA & $\begin{array}{l}\text { No steroid } \\
\text { use }\end{array}$ \\
\hline $\begin{array}{l}\text { Hamano } \\
\text { et al. }{ }^{10}\end{array}$ & F & 50 & Biopsy & CBD & $\begin{array}{c}\text { Abdominal } \\
\text { pain, jaundice }\end{array}$ & $\begin{array}{l}\text { Mass with long } \\
\text { CBD narrowing }\end{array}$ & NA & Initial & No & $\begin{array}{c}122 \\
/ 1,711\end{array}$ & $\begin{array}{l}\text { Response } \\
\text { on steroids }\end{array}$ \\
\hline \multirow[t]{5}{*}{ Zen et al. ${ }^{9}$} & M & 59 & Resection & $\mathrm{IBD}$ & NA & $\begin{array}{l}\text { Mass with irregular } \\
\text { stricture }\end{array}$ & $+++^{\mathrm{a}}$ & Initial & No & NA & NA \\
\hline & M & 79 & Resection & $\mathrm{IBD}$ & NA & $\begin{array}{l}\text { Mass with irregular } \\
\text { stricture }\end{array}$ & $+++^{a}$ & Initial & No & NA & NA \\
\hline & M & 56 & Resection & $\mathrm{IBD}$ & NA & $\begin{array}{l}\text { Mass with irregular } \\
\text { stricture }\end{array}$ & $+++^{a}$ & Initial & LN & NA & NA \\
\hline & M & 64 & Resection & $\mathrm{IBD}$ & NA & $\begin{array}{l}\text { Mass with irregular } \\
\text { stricture }\end{array}$ & $+++^{a}$ & Initial & LN & NA & NA \\
\hline & M & 67 & Resection & Left HD & NA & $\begin{array}{l}\text { Mass with irregular } \\
\text { stricture }\end{array}$ & $+++^{a}$ & Initial & No & NA & NA \\
\hline
\end{tabular}

HPF, high power field; M, male; LN, lymph node; IBD, intrahepatic bile duct; F, female; NA, not available; CBD, common bile duct; HD, hepatic duct. aThe number of IgG4-immunopositive plasma cells is not counted; semiquantitative score is used instead. 
most reported cases were identified as long segment smooth narrowing of the bile ducts. ${ }^{14}$ Table 1 summarizes clinical, radiological and pathologic characteristics of localized mass-forming IgG4-related cholangitis cases that have been reported to date. Besides our case, all except one presented as hilar or intrahepatic mass accompanied by biliary stricture. ${ }^{8-10}$ Since these lesions had often been misdiagnosed as cholangiocarcinoma, many patients had undergone unnecessary resection of liver. IgG4-RD could be diagnosed only after histopathologic examination of the resected specimen, which showed numerous IgG4+ plasma cell infiltrates. ${ }^{9,15,16}$

The case presented here clearly showed a mass lesion in both imaging studies and resected specimen without accompanying biliary stricture. Radiologists also had no doubt that the lesion was malignancy since it was not only a well-defined isolated mass but also it had characteristic features of malignant lesion in many imaging modalities; arterial phase of CT revealed enhancement and the lesion showed high signal intensity on diffusion restriction phase and low intensity on ADC map phase of MRI. Furthermore, the patient lost $3 \mathrm{~kg}$ of his weight for 10 days, a suspicious sign of malignancy. Multiple enlarged lymph nodes in our case were also regarded as metastasis from cholangiocarcinoma until the aspiration biopsy proved otherwise. Despite the negative result of aspiration biopsy of the lymph node, hilar cholangiocarcinoma was still suspected, considering the result as false-negative. However, a possibility of nodal manifestation of IgG4-RD should have been considered in this case because systemic nodal metastasis is an unusual finding for cholangiocarcinoma. ${ }^{17}$

Clinical diagnostic criteria to aid detection of IgG4-related sclerosing cholangitis were suggested by a Japanese group. ${ }^{18}$ The criteria included four items: (1) characteristic biliary imaging findings, (2) elevation of serum IgG4, (3) coexistence of other IgG4-related disease, and (4) histopathological features. To make definite diagnosis, thorough investigation of clinical, radiologic, laboratory and histopathologic examination is essential; however, all of these examinations are not always performed. Although there are some radiologic characteristics which help distinguish IgG4-related cholangitis from primary sclerosing cholangitis, it is still difficult to exclude hilar cholangiocarcinoma by image alone. Likewise, serum IgG4 level alone is not helpful since its sensitivity and specificity are not high. ${ }^{19}$ Some of IgG4related cholangitis cases showed only minimally elevated serum IgG4 level. ${ }^{10}$ Histologic confirmation is necessary for definite diagnosis in this regard. Biopsy seems to be superior to brush cytology because some cases revealed false-positive atypical cells in brush cytology but resection specimen consisted of IgG4+ plasma cells and fibrosis only. ${ }^{20}$ Both quantity and quality of the biopsy are crucial for representing the entire lesion and immunohistochemical staining for $\operatorname{IgG}$ and $\operatorname{IgG} 4$ is required for an accurate diagnosis.

In summary, we report an unusual case of localized mass-forming IgG4-related cholangitis that mimicked hilar cholangiocarcinoma in the initial diagnostic approach. Since isolated massforming IgG4-RD without biliary stricture is extremely rare, exclusion of malignancy can be difficult without histopathologic confirmation. Also, the IgG4-related cholangitis in this patient later progressed to the most extensive form of systemic disease reported to date. Although IgG4-related cholangitis is rare, it should be considered in differential diagnosis of a solitary mass in the biliary tree that mimics cholangiocarcinoma, especially when it is accompanied by any evidence of systemic manifestation, including multiple lymphadenopathy and suspicious extrapancreatic organ involvement.

\section{Conflicts of Interest}

No potential conflict of interest relevant to this article was reported.

\section{REFERENCES}

1. Zen Y, Nakanuma Y. IgG4-related disease: a cross-sectional study of 114 cases. Am J Surg Pathol 2010; 34: 1812-9.

2. Deshpande V, Zen Y, Chan JK, et al. Consensus statement on the pathology of IgG4-related disease. Mod Pathol 2012; 25: 1181-92.

3. Stone JH, Zen Y, Deshpande V. IgG4-related disease. N Engl J Med 2012; 366: 539-51.

4. Mahajan VS, Mattoo H, Deshpande V, Pillai SS, Stone JH. IgG4-related disease. Annu Rev Pathol 2014; 9: 315-47.

5. Chang WI, Kim BJ, Lee JK, et al. The clinical and radiological characteristics of focal mass-forming autoimmune pancreatitis: comparison with chronic pancreatitis and pancreatic cancer. Pancreas 2009; 38: 401-8.

6. Matsumoto I, Shinzeki M, Toyama H, et al. A focal mass-forming autoimmune pancreatitis mimicking pancreatic cancer with obstruction of the main pancreatic duct. J Gastrointest Surg 2011; 15: 2296-8.

7. Naitoh I, Nakazawa T, Hayashi K, et al. Clinical differences between mass-forming autoimmune pancreatitis and pancreatic cancer. Scand J Gastroenterol 2012; 47: 607-13.

8. Deshpande V, Sainani NI, Chung RT, et al. IgG4-associated cholan- 
gitis: a comparative histological and immunophenotypic study with primary sclerosing cholangitis on liver biopsy material. Mod Pathol 2009; 22: 1287-95.

9. Zen Y, Harada K, Sasaki M, et al. IgG4-related sclerosing cholangitis with and without hepatic inflammatory pseudotumor, and sclerosing pancreatitis-associated sclerosing cholangitis: do they belong to a spectrum of sclerosing pancreatitis? Am J Surg Pathol 2004; 28: 1193-203.

10. Hamano H, Kawa S, Uehara T, et al. Immunoglobulin G4-related lymphoplasmacytic sclerosing cholangitis that mimics infiltrating hilar cholangiocarcinoma: part of a spectrum of autoimmune pancreatitis? Gastrointest Endosc 2005; 62: 152-7.

11. Hamano H, Kawa S, Horiuchi A, et al. High serum IgG4 concentrations in patients with sclerosing pancreatitis. N Engl J Med 2001; 344: 732-8.

12. Kawano M, Saeki T, Nakashima H, et al. Proposal for diagnostic criteria for IgG4-related kidney disease. Clin Exp Nephrol 2011; 15: 615-26.

13. Novotný I, Dítě P, Trna J, Lata J, Husová L, Geryk E. Immunoglobulin G4-related cholangitis: a variant of IgG4-related systemic disease. Dig Dis 2012; 30: 216-9.
14. Deshpande V. IgG4-related disease of the gastrointestinal tract: a 21st century chameleon. Arch Pathol Lab Med 2015; 139: 742-9.

15. Miki A, Sakuma Y, Ohzawa H, et al. Immunoglobulin g4-related sclerosing cholangitis mimicking hilar cholangiocarcinoma diagnosed with following bile duct resection: report of a case. Int Surg 2015; 100: 480-5.

16. Graham RP, Smyrk TC, Chari ST, Takahashi N, Zhang L. Isolated IgG4-related sclerosing cholangitis: a report of 9 cases. Hum Pathol 2014; 45: 1722-9.

17. Cheuk W, Yuen HK, Chu SY, Chiu EK, Lam LK, Chan JK. Lymphadenopathy of IgG4-related sclerosing disease. Am J Surg Pathol 2008; 32: 671-81.

18. Ohara H, Okazaki K, Tsubouchi $\mathrm{H}$, et al. Clinical diagnostic criteria of IgG4-related sclerosing cholangitis 2012. J Hepatobiliary Pancreat Sci 2012; 19: 536-42.

19. Lazaridis KN. Sclerosing cholangitis epidemiology and etiology. J Gastrointest Surg 2008; 12: 417-9.

20. Chung DT, Tang CN, Lai EC, Yang GP, Li MK. Immunoglobulin G4-associated sclerosing cholangitis mimicking cholangiocarcinoma. Hong Kong Med J 2010; 16: 149-52. 